\title{
A taxonomy of five elements to evaluate a secure network
}

\author{
E. Portillo, J. Carracedo \\ Univ. Politécnica de Madrid (DIATEL-UPM) \\ Dpto. Ing. y Arq. Telemáticas \\ EUIT. Telecomunicación / UPM \\ Ctra. de Valencia, Km. 7 / E-28031 Madrid Spain \\ Ph.: +34-1-336 7820 Fax: +34-1-336 7817 \\ E-mail: \{Portillo,Carracedo\}@diatel.upm.es
}

\begin{abstract}
When treating technological topics, the approach may be either more instrumental focusing on the tools and elements of the discipline or more methodological studying the way in which these tools are used and fit an organizational or socio-technical reality. In order to enhance the study of the complexity of computer security, and using the authors' earlier classification of security elements (Portillo, 1996), we have enlarged this traditional division to include the identification of five branches or aspects that are present in every secure system. In the complex and ever-changing environment of Information Technologies, this taxonomy establishes a model that can help users and experts to design, evaluate, and operate secure systems. These five topics are: technological elements, multilevel issues, placement of security services, security policy and socio-technical aspects. Finally, a brief panoramic analysis is provided, in which, the parallel historical evolution of the five branches, and their mutual influences can be observed.
\end{abstract}

\section{Keywords}

Security of data, Computer networks, Internetworking, Social aspects of automation, Modelling

\section{INTRODUCTION}

Cryptography has long been the foundation of information protection. In traditional cryptography, usually known as secret key cryptography or single-key cryptography, sender and receiver share only one key that is used both to cipher and to decipher. With this method, the most sensitive operation is the transporting of the key to each interlocutor and its adequate storage. In case of interception of the key, cryptography looses its utility and 
messages can be deciphered not only by its receiver but also by any interceptor of the key. In the 70's, the public key (or double key) cryptographic systems appeared (Diffie, 1976 and Rivest, 1978). In this systems, two complementary operations (such as cipher and decipher, or signing and verifying a signature) are taken on by using two different elements of a compound key. One element is kept secret by the user and is called the private element and the other is sent to his counterparts, and called the public element. In this system, the private component of the key (used to sign or to decipher) need not travel at all. The interception of the public component of the key is no longer a danger because this component is only useful to cipher and to verify a signature, and never to decipher or sign a message.

Nevertheless, a system of public keys is easily attacked by supplanting a personality: when someone gets a key and thinks that it belongs to a user when it has really been generated by a suplanter, then the person who really generated the key can be impersonated by the other one. Although this problem is resolved, initially, by using certificates there is no system that offers complete security, especially when it is not used properly. It has been demonstrated that the use of a cryptographic algorithm to protect information is not enough by itself, but requires the study of how the different protection measures are integrated into the system taken in its totality. In addition, as is discussed further on, organizational and methodological issues must be taken into account.

\section{SOME CONCERNS ON THE USE OF CRYPTOGRAPHY}

Cryptography does not constitute a secure system by itself. A key can be very difficult to break and still be very poorly storaged. If someone steals this key, he will be able to intercept the communications despite the sophistication and strength of the cipher algorithm. Regarding this, it is usually said that 'the chain is always broken by the weakest link' and there are people that add that this was never so true as it is in the case of modern computer networks. For example, very well designed secure systems are frequently managed by people who lack the necessary preparation or time for its correct operation. Under these circumstances, managers fall into errors of system configuration and operational procedures that finally produce cracks in the system security. This is the origin of most of intrusion -hacking- incidents.

It is now evident that security is holistically evolved in every element of the system and its components. The study of the security of each element and the relationship between elements is vital to the achievement of security levels for systems higher than one prefigured security level.

\section{A TAXONOMY OF SECURE SYSTEMS}

When we evaluate, for example, an electronic mail system, we will have to determine whether or not each user and every one of their messages needs the same protection. Perhaps some of them need a cipher process to protect confidentiality while others just need a digital signature. Additionally, it must be born in mind that security is sometimes costly (for example, when specific hardware such as smartcards are used) and that in a security plan for an entire organization, it can be costly to provide the maximum security level to every user and every document. Thus we are faced with what it is known a multilevel security issue: in a complex system where a great variety of needs exist, the security requirements are very different among the different users, documents, links and servers. Therefore, they must be classified into several security levels. 
Variations are also present when we look at the specific moments when the information is protected. In the discussion of cryptography, we have seen an example of intuitive protection called end-to-end protection, in which, the user codifies a message before sending it and the receiver decodifies it. But there are also some situations in which other procedures are needed. Let us consider a mail system that is interconnected into a wider network, for example, a company that uses a world message service. If we want to guarantee the delivery of every message in the safest possible way and we also want to insure accountability in the case of possible loss or delay we must add a utility with which to establish secure accounting at the interconnection point, in order to produce evidence regarding the moment each message is delivered. This is a security element installed inside the network, different from any end-to-end element. Moreover, this type of intermediate element is often used to produce redundant security. This is the case of the firewalls (Wack, 1995) that are used in corporate networks to isolate them from the outside or that are installed inside the company network to establish subnetworks of different security levels. It can be concluded that when designing or reforming any security system a series of decisions regarding the placement of security services must be taken.

Even when the technological elements -with simple or multiple protection levels- have been chosen, and the places on the network on which they are to be installed are decided, all are useless if they are not used in the correct order and according to the proper procedures, e. g. storing the keys properly, renewing them periodically, closing a console when the user is absent, etc. This list of rules is called the security policy, and depending on the environment, it can consist of anything from a series of simple, almost informal rules up to a complex list of rules and recommendations, some of them in less formal language and others that are codified and applied by the systems in a automatic way.

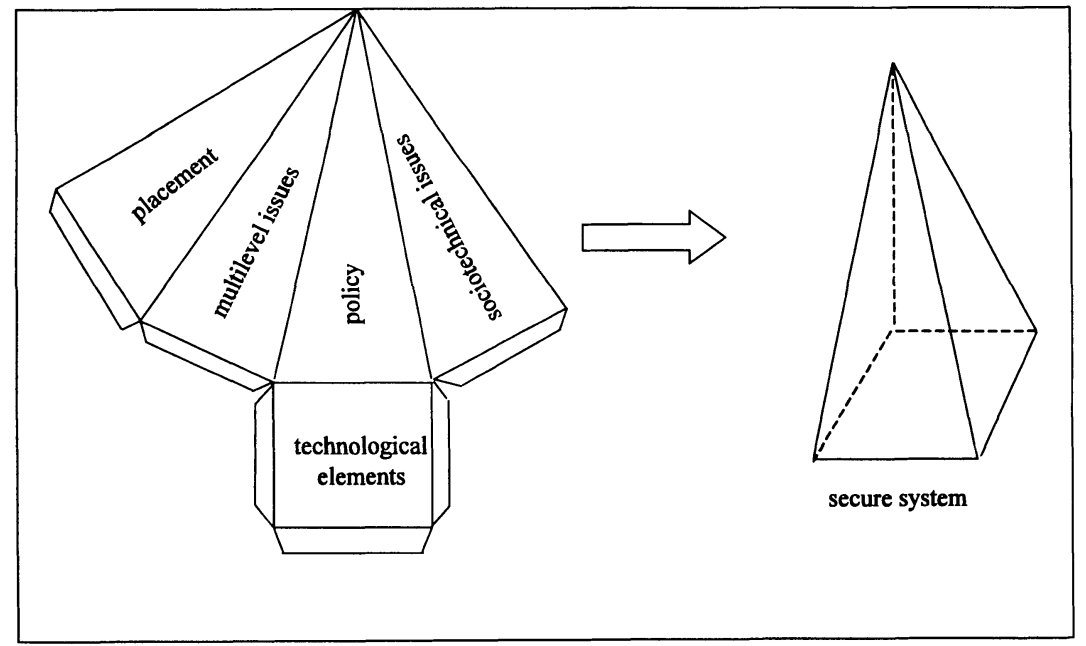

Figure 1 Five aspects to evaluate in every secure system.

Last but not least, there are some socio-technical and organizational issues that are related to the operation of any information system: the security policy must not only be correctly and clearly published but should also involve the users in its elaboration, in as much as they are better informed than anyone else about the different risks and values of each piece of 
information. Another element to be taken into account is the existance of legal restrictions, the interest of the public powers and the defense of the rights of the individuals.

It is evident that users, managers and security experts must cooperate in the continuous upgrading of the security of the system just as they do in other modern applications of total quality management (Ishikawa, 1995). The upgrading of security policy is one of the main tasks of these multidisciplinary groups, as well as the taking into account of the constrains of regulations from inside and outside of the organization.

In summary, a total of five outstanding aspects (see Figure 1) must be considered when developing or using secure systems. They make up five fields for which specific engineering solutions must be provided. They are the following:

- basic technological elements: specific hardware, cryptographic algorithms, etc.

- multilevel issues

- placement of security services

- security policy

- socio-technical, legal and organizational aspects

\section{EVOLUTION OF SECURE SYSTEMS}

The following paragraph addresses the manner in which the continuous advances in computer security have incorporated new elements of the five aspects, and the way in which the development of each aspect is often parallel due to the interdependence of the different areas. It is evident that secure systems quickly become more and more complex. When studing the five aspects, we can observe how each of them grows in complexity as represented in Figure. 2.

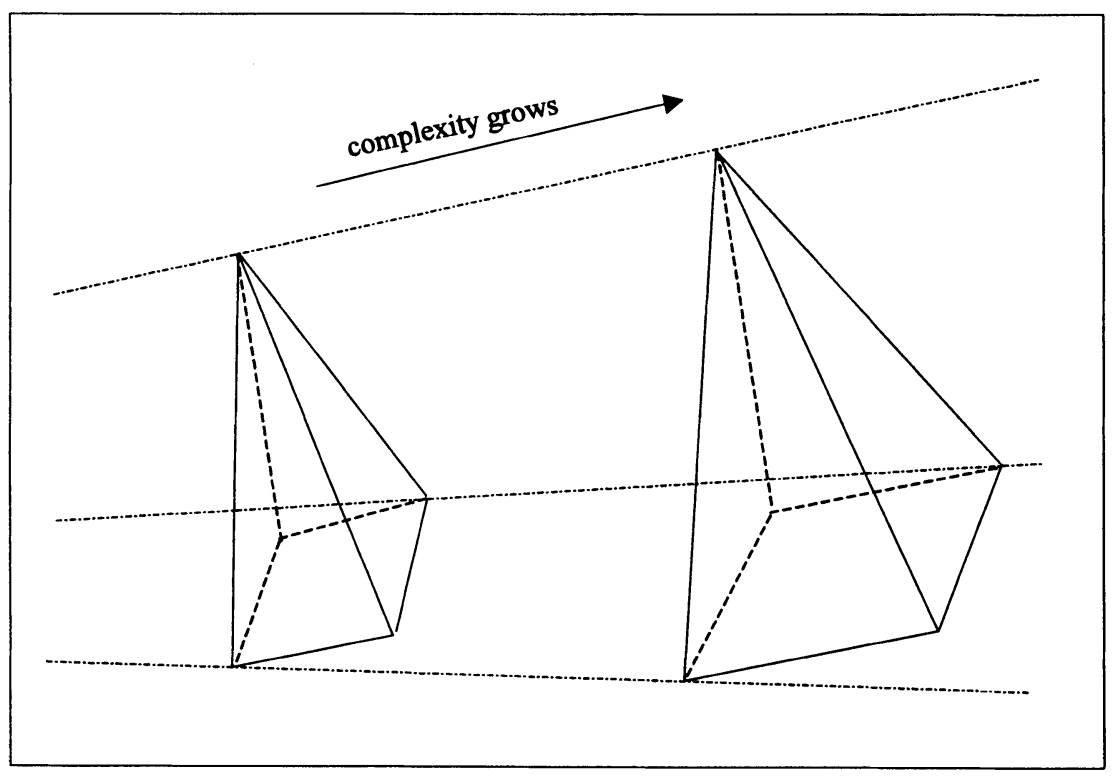

Figure 2 Complexity grows in every aspects and in the system as a whole. 
Many basic technological elements, both hardware and algorithms, have been incorporated into secure systems. Among the most important ones, we can point out: single key cipher algorithms, public key cipher algorithms and public key certificates. Removable hardware elements make excellent storage for cryptographic material and, additionally, provide the user with a subjective sense of security when they remove them from the system. This kind of removable element has evolved extremely rapidly from the magnetic tape card to the smart card and, more recently to the PCMCIA card. Some new elements should be mentioned as being useful for more specialized and sophisticated security services: e.g. CD-WORMs for non-repudiation or blind signature for anonymity (Chaum, 1985).

Regarding placement, one must remember that in the early days of mainframes and dumb terminals interconnected in a star topology, the most common security device was a secret key cipher that was placed on either side of the link joining two buildings or installations within the same organization. In these systems security measures were mainly applied to links.

In addition, the end-to-end security is included in each new available application (E-mail, remote terminal access, Web, etc.). The rise of package networks (x.25 or IP) has lead to the appearance of filtering routers which protect servers from unauthorized access by filtering those packages that do not have the correct origin. These are the predecessors of more complex firewalls with several elements capable of authenticating a machine, a user and/or a remote service before giving access to a sub network. Another interesting concept in relation to the security service placement is the proxy application. Using this procedure a group of machines within a subnetwork which is not connected to a given service can receive this service by means of a server that works as an intermediary, allowing the use of these services to be controlled centrally.

The combination of firewalls and proxy application applied to complex corporate networks has lead to the concept of intranet which is the secure interconnection of corporate networks in such a way that any given network can remain beyond the reach of global Internet (even the addresses of these machines are not registered globally, but only on a local level). Each machine then receives several global services through proxy applications. If, in addition, service duplication is used, different users can even receive personalized responses when they apparently access the same information from the same server.

Regarding the difficulties of multilevels, the initial approach was to create networks for each department (or group of users) that were completely isolated from each other. Although this approach is still employed in cases of critical services, it is now more common for users with different security levels to share the same channels. In order to do this, CIPSO has been developed. Another alternative is to classify users and services directly by name or specific attributes of authorization (like Digital VMS Right Identifiers).

Lastly, today's challenge is global interoperability in an open world in which diverse organizations adhere to their own policies which they have codified at different levels of sensitivity.

Early informal security policies, were lists of "dos and don'ts" such as "make security copies every week" or "lock the computer center when you leave". However, global networks have created different compendiums: diverse FAQs (aimed at hackers or administrators, which are freely distributed or supposedly restricted) and patch lists which ought to be applied (with or without the blessing of the CERT). With the arrival of multi-user systems, a set of passwords and obligatory access are established in such a way that the rules applying to the reading or modification of each resource in the system can be codified. If the system includes cryptography the security policy should include such things as validity periods and procedures for their substitution. Public key certificates have made the distribution of keys more versatile as well as more complex (rules for revoking keys and checking their validity). The different uses which can be made of a given key (authentication, access control, 


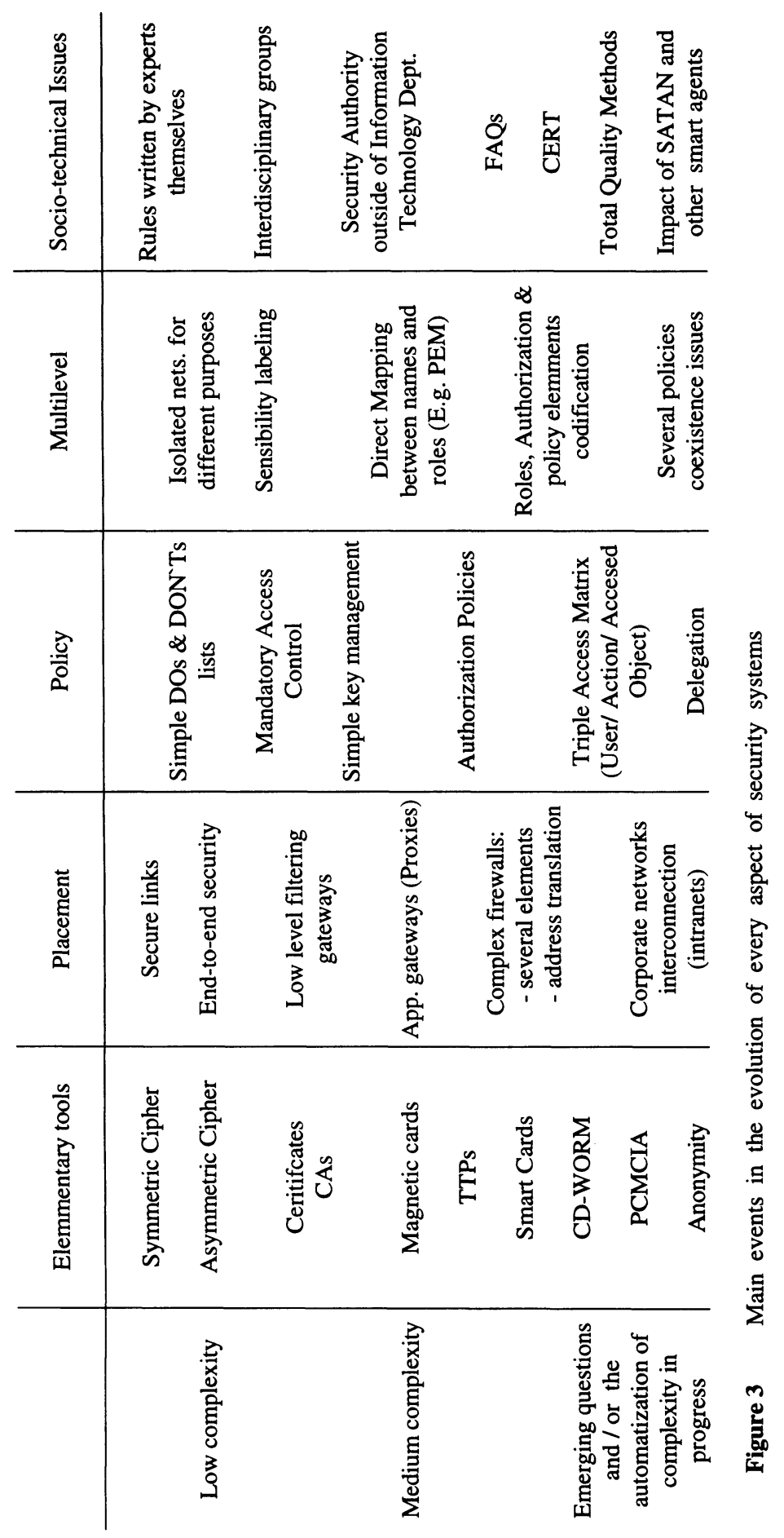


validation of a transaction) require the development of authorization policies in order to determine unambiguously the destination for which a key was certified.

Finally, one of the most complex problems is the establishment of delegation policies in which an agent receives temporary authorization to carry out an operation for the real owner of the right to use. The problem of delegation and the use of roles in order to control access based on names are currently under study.

It is also necessary to study the socio-technological environment surrounding the implementation and operation of secure systems. We will focus on the procedures by which users collaborate in the elaboration of these policies, the distribution of the different functions of computer security, the economic quantification of the risks and costs of protection and any other issues that deal with the relation between security technology and the organizational and economic realities of companies and institutions. Initially, computer experts dictated policy to non-expert users. Later the figure of the security expert developed and finally that of the auditor . An auditor who should, ideally, be outside the computer department which he is responsible for auditing. Unfortunately, this condition remains unfulfilled in many organizations. In order to optimize the collaboration between users and experts, interdisciplinary groups have been created although their methods and results are not always clear One interesting approach is the use of groupwork techniques, Total Quality Circles and the discussion of Technology Transfer.

In closing we would like to indicate that the revolution resulting from programs such as SATAN, which scan a system or a subnetwork in search of any and all weak points. These ambivalent programs that can be used either by a security expert to improve prevention or by an attacker who wants to take advantage of a weak point are authentic determiners of the security level of a system. Unfortunately for legitimate administrators, these scanning agents that can help in detection result in the extremely rapid evolution ofdifferent versions of operating systems and application programs while leading in the best case to a very dynamic equilibrium between errors detected and errors resolved.

Using the main events in network security developement, Figure 3 shows a comparison of the evolution of the five above mentioned aspects. The items exposed have been selected from various reference works and specially from Scheneier (1994).

As we have seen, the core of a secure system can be observed from five different points of view. Each one of these 'views' consists of a set of aspects and elements that must be taken into account when describing the whole system. The metaphor of the pyramid used in this paper illustrates the fact that the union of all the elements encompasses the system itself. We have also tried to emphasize that the growing complexity of the system affects each element within it simultaneosly. Many agents with different levels of expertise are involved in the issue of network security: security experts, designers, managers, end users...the authors believe that the proposed model can be useful in raising the level of awareness of network security issues of these agents as well as in the description and teaching of the structure and behavior of these systems.

Further work is needed in the formalization of these contributions in order to generate a valid methodology to design or to evaluate a secure system. 
CIPSO (1993) Security Label. MIL-STD 2045-48501, also known as Common IP Security Option. (TSIG-CIPSO), Dpt. of Defense USA.

Chaum, D. (1985) Security without Identification: Card Computers to make Big Brother Obsolete. Communications of the ACM, 28(10):1030-1044. ACM.

Diffie, W. and Hellman, M. E. (1976) New Directions in Cryptography, IEEE Transactions on Information Theory, v. IT-22, n. 6, Nov. 1976, pp. 644-654.

Ishikawa, K. (1995) Quality Control: The Japanese Way, Prentice-Hall.

Portillo, E and Carracedo J.(1996), Seguridad en redes complejas: el caso Internet in proceedings of I Congreso de la Asociación de Usuarios Internet, Madrid. (in spanish)

PEM (1993) Privacy Enhancement for Internet Electronic Mail (PEM), Internet RFCs 14211424.

Rivest, R. L., Shamir, A.and Adelman, L. M. (1978) A Method for Obtaining Digital Signatures and Public-Key Cryptosystems, Communications of the ACM, v. 21, n. 2, 1978, pp. 120-126.

Schneier, B. (1994) Applied Cryptography. John Wiley and Sons, Inc..

TNI (1987) Trusted Network Interpretation (TNI) of the Trusted Computer System Evaluation Criteria (TCSEC), usually known as The Red Book, NCSC-TG-005 Version 1, 1987.

J. P. Wack J. P.and L. J. Carnahan (1995) Keeping Your Site Comfortably secure: An Introduction to Internet Firewalls. NIST Special Publication 800-10 National Institute of Standars and Technology. http://csrc.nist.gov/nistpubs/800-10/.

Professor Eloy Portillo was born on 1964, he holds a MSc. on Theoretical Physics by the Autonomous University of Madrid (UAM, Jun. 87). He has worked as a junior engeneering in computer networks at the UAM Computer Center; Head of Networking (1990-1992). On 1992, lecturer on Telematics at his present Department (DIATEL-UPM) at the Technical University of Madrid (Univ. Politécnica de Madrid UPM) and researcher student on the area of Network Security in which he is preparing the degree of $\mathrm{PhD}$ under the direction of Justo Carracedo PhD. and Ana Gómez PhD.

Dr. Justo Carracedo holds a MSc. on Telecommunications and a PhD. on Computer Science, both at Technical University of Madrid (Universidad Politécnica de Madrid - UPM). He is professor at the Telematic Engineering Department (DIATEL) at the UPM, where he leads the research group on Network Security. He is also working on Distributed Applications and Formal Description Techniques. He has participated in many national and European projects and cooperates on the efforts of standardisation while taking part on several ISO national committees. He is a permanent counsellor of the Spanish Ministry for the Public Administrations. 